\title{
AN EXTENSION OF THE GERBER-BÜHLMANN-JEWELL CONDITIONS FOR OPTIMAL RISK SHARING
}

\author{
BY \\ MAREK KALUSZKA
}

\begin{abstract}
We provide necessary and sufficient conditions for optimality of mutual contracts for risk sharing under constraints on premiums or utility functions of participants of the agreement. These conditions are an extension of those of the Borch, Gerber and Bühlmann-Jewell ones. Some applications to optimal insurance contracts, optimal dividend sharing and optimal reinsurance are given.
\end{abstract}

\section{KEYWORDS}

Insurance, Limited insurance, Reinsurance, Sharing risks, Dividends.

\section{INTRODUCTION}

A mutual agreement of $n$ companies will be considered in the paper. Let $X_{i}$ denote a wealth which is reported to redistribution by $i$ th participant of the agreement. We assume that $X_{i}$ is a random variable defined on a given probability space $(\Omega, S, \mathbb{P})$, which may take negative values with positive probability. Let $X=X_{1}+\ldots+X_{n}$ be the global wealth. Throughout the paper, the notation $X=Y$ and $X<Y$ means $\mathbb{P}(X=Y)=1$ and $\mathbb{P}(X<Y)=1$, respectively. We write $R_{i}$ for a part of the global wealth redistributed to $i$ th participant of the agreement. The sequence of random variables $\left(R_{i}\right)=\left(R_{i}, \ldots, R_{n}\right)$ will be called a sharing rule if the clearing condition is satisfied: $\sum_{i=1}^{n} R_{i}=\sum_{i=1}^{n} X_{i}$. For instance, in reinsurance $X_{1}=-Y, X_{2}=0, R_{1}=R-Y-P$, and $R_{2}=P-R$, where $Y$ denotes the aggregate claim amount of the first insurer, $R=R(Y)$ denotes a compensation paid by the reinsurer, and $P$ stands for a reinsurer's premium.

How to determine optimal rules for sharing risks and constructing reinsurance treaties? The classical results are due to de Finetti (1940). De Finetti derived relative retention levels which have simple forms by considering the insurer's net (of reinsurance) profit from the portfolio at the end of a given time period. He then minimized the variance of this profit subject to its expected value being fixed. See also Bühlmann (1996), Section 5.2, and Ammeter et al. (1959) for a review of ealier works of Medolaghi, Ottaviani and others. 
In the seminal paper of Borch (1962) another method is presented. Borch adapted the von Neumann-Morgernstern utility theory to actuarial science and proposed criteria based on the concept of utility function For an excellent overview of the expected utility theory, we refer the reader to Panjer et al. (1998) and Gollier (2001). We now provide the essential points of Borch's results. More details, and proofs, can be found in Borch (1974, 1990). Assume preferences of $i$ th participant can be described by a utility function, say $u_{i}$. The common examples are the

- exponential utility function with index $\alpha u_{\alpha}(x)=\frac{1}{\alpha}\left(1-e^{-\alpha x}\right), x \in \mathbb{R}, \alpha>0$; this function yields a constant risk aversion,

- logarithmic utility function $u(x)=\ln x, x>0$; the risk aversion is decreasing with wealth,

- power utility function of the first kind, $u(x)=\frac{a^{c+1}-(a-x)^{c+1}}{(c+1) a^{c}}, x<a, a, c>0$; the risk aversion increases with wealth,

- power utility function of the second kind, $u(x)=\frac{1}{c}\left(x^{c}-1\right), x>0,0<c<1$; the risk aversion is a decreasing function of wealth.

Borch (1962) proposed to choose a Pareto-optimal risk sharing as an optimal one. We say that the rule $\left(R_{i}\right)$ is Pareto-optimal if there is no rule $\left(\hat{R}_{i}\right)$ such that $\mathbb{E} u_{i}\left(\hat{R}_{i}\right) \geq \mathbb{E} u_{i}\left(R_{i}\right)$ for all $i$ with strict inequality for some $i$. It is well known that if the rule $\left(R_{i}\right)$ is a solution of the following constrained optimization problem

$$
\max \sum_{i=1}^{n} k_{i} \mathbb{E} u_{i}\left(R_{i}\right) \text { s.t. } \sum_{i=1}^{n} R_{i}=X,
$$

with positive reals $\left(k_{i}\right)$, it is Pareto-optimal (see e.g. Gerber, 1979, p. 90, for a geometric justification). Borch showed that $\left(R_{i}\right)$ solves the problem (1.1) if and only if the Borch condition holds: $k_{i} u_{i}^{\prime}\left(R_{i}\right)=k_{n} u_{n}^{\prime}\left(R_{n}\right)$ for all $i$ (for the proof see e.g. Gerber and Pafumi, 1999). Denote by $D_{f}$ the domain of the function $f$ and suppose the support of $X$ is contained in $D_{u_{1}}+\ldots+D_{u_{n}}=\left\{x_{1}+\ldots+x_{n} ; x_{i} \in D_{u_{i}}\right\}$. From the Borch condition it follows that if $\left(u_{i}\right)$ are differentiable and strictly concave and if $u_{i}^{\prime}\left(D_{u_{i}}\right)=(0, \infty)$ for all $i$, then the solution of the problem (1.1) is given by

$$
R_{i}(x)=u_{i}^{*}\left(\frac{k_{n}}{k_{i}} u_{n}^{\prime}\left(h^{*}(x)\right)\right), i=1, \ldots, n,
$$

where $u_{i}^{*}$ and $h^{*}$ denotes, respectively, the inverse function of $u_{i}^{\prime}$ and

$$
h(x)=\sum_{i=1}^{n} u_{i}^{*}\left(\frac{k_{n}}{k_{i}} u_{n}^{\prime}(x)\right)
$$

(cf. Pesonen, 1984, Wyler, 1990). Borch's result was extended by Deprez and Gerber (1985) who considered minimization of $\sum_{i=1}^{n} \Pi_{i}\left(R_{i}\right)$ subject to $\sum_{i=1}^{n} R_{i}=X$, where $\Pi_{i}$ is a convex and Gâteaux differentiable functional. It was proved that 
$\left(R_{i}\right)$ minimizes the functional $\sum_{i=1}^{n} \Pi_{i}\left(R_{i}\right)$ if and only if the extended Borch condition is satisfied: $\Pi_{i}^{\prime}\left(R_{i}\right)=\Pi_{n}^{\prime}\left(R_{n}\right)$ for each $i$, where $\Pi_{i}^{\prime}$ stands for the Gâteaux differential of $\Pi_{i}$. Unfortunately, there is a well known drawback of the Borch method illustrated by the following example.

\section{Example}

1.1. Assume that the $i$ th company uses the exponential utility function with an index $a_{i}$. A solution of the problem

$$
\max \sum_{i=1}^{n} \frac{k_{i}}{a_{i}} \mathbb{E}\left[1-\exp \left(-a_{i} R_{i}\right)\right] \text { s.t. } \sum_{i=1}^{n} R_{i}=X
$$

is the quota rule $R_{i}=\left(a / a_{i}\right) X+b_{i}$, where $1 / a=\sum_{i=1}^{n} 1 / a_{i}$ and $b_{i}$, called a side payment, depends on $k_{i}$ (see e.g. Gerber and Pafumi, 1999). Because the side payments must sum to zero, there are some companies making payments to others even when all losses are zero.

To remove this disadvantage Gerber (1978) and Bühlmann and Jewell (1979) proposed to include side constraints on $R_{i}$. Define

$$
\mathcal{R}_{L}^{U}=\left\{\left(R_{i}\right) ; \sum_{i=1}^{n} R_{i}=X, L_{i} \leq R_{i} \leq U_{i}, i=1,2, \ldots, n\right\}
$$

in which $L=\left(L_{i}\right), U=\left(U_{i}\right)$, and $L_{i} \leq U_{i}$ are arbitrary random variables with values in $[-\infty, \infty]$. Gerber (1978) proved that a rule $\left(R_{i}\right)$ solves the problem

$$
\max \sum_{i=1}^{n} k_{i} \mathbb{E} u_{i}\left(R_{i}\right) \text { s.t. }\left(R_{i}\right) \in \mathcal{R}_{L}^{U},
$$

where $U=(\infty)$ and $L=\left(X_{i}-c_{i}\right)$ with nonnegative reals $\left(c_{i}\right)$ if and only if $\left(R_{i}\right)$ satisfies the Gerber condition:

$$
R_{i}=X_{i}-c_{i} \text { whenever } k_{i} u_{i}^{\prime}\left(R_{i}\right)<\max \left\{k_{j} u_{j}^{\prime}\left(R_{j}\right) ; j=1, \ldots, n\right\},
$$

(see also Gerber, 1979). In this framework, company $i$ is not willing to pay more that $c_{i}$ toward losses of other companies.

Bühlmann and Jewell (1979) stated that a rule $\left(R_{i}\right)$ is a solution of the problem (1.5) with $L=(0)$ if and only if the Bühlmann-Jewell condition holds: there exist a positive random variable $\Lambda$ and positive constants $\left(k_{i}\right)$ such that for $i=1,2, \ldots, n$

$$
\begin{aligned}
& k_{i} u_{i}^{\prime}\left(R_{i}\right)=\Lambda \text { if } 0<R_{i}<U_{i}, \\
& k_{i} u_{i}^{\prime}\left(R_{i}\right) \leq \Lambda \text { if } \quad R_{i}=0 \\
& k_{i} u_{i}^{\prime}\left(R_{i}\right) \geq \Lambda \text { if } \quad R_{i}=U_{i} .
\end{aligned}
$$

The Bühlmann-Jewell constraint $L=(0)$ can be explained as follows: company $i$ is not ready to lose more than its total wealth in the worst. For $L=(0)$ and $U=(\infty)$, they also found the following explicit solution of (1.5): 


$$
R_{i}(x)=\left[u_{i}^{*}\left(\frac{k_{n}}{k_{i}} u_{n}^{\prime}\left(h^{*}(x)\right)\right)\right]_{+}, x \geq 0, i=1, \ldots, n,
$$

in which $u_{i}^{*}$ and $h^{*}$ denotes, respectively, the inverse function of $u_{i}^{\prime}$ and

$$
h(x)=\sum_{i=1}^{n}\left[u_{i}^{*}\left(\frac{k_{n}}{k_{i}} u_{n}^{\prime}(x)\right)\right]_{+}, x \geq 0 .
$$

In actuarial literature one can find a few methods of choosing coefficients $\left(k_{i}\right)$ in (1.2) or (1.6). The individual rationality condition says that the coefficients $\left(k_{i}\right)$ are acceptable if $\mathbb{E} u_{i}\left(R_{i}\right) \geq \mathbb{E} u_{i}\left(X_{i}\right)$ for all $i$. There are many Pareto-optimal rules violating this condition. Furthermore, the condition may not provide exactly one solution. For instance, from the extended Borch condition it follows that the solution of the problem

$$
\min \sum_{i=1}^{n} k_{i} \mathbb{D}^{2} R_{i} \text { s.t. } \sum_{i=1}^{n} R_{i}=X
$$

is the quota rule $R_{i}=\left(k / k_{i}\right) X+b_{i}$, where $1 / k=\sum_{i=1}^{n} 1 / k_{i}$, and $\left(b_{i}\right)$ are any reals such that $b_{1}+\ldots+b_{n}=0$. In what follows, $\mathbb{D}^{2} Y$ denotes the variance of random variable $Y$ and $\mathbb{D} Y$ denotes the standard deviation. The condition of individual rationality implies that $0<k / k_{i} \leq \mathbb{D} X_{i} / \mathbb{D} X$ for all $i$. Clearly, $\mathbb{D} X \leq \sum_{i=1}^{n} \mathbb{D} X_{i}$ so there exist a lot of rules satisfying this condition.

Another proposition one can find in Bühlmann (1984). He suggested to choose $\left(k_{i}\right)$ according to the nonprofit condition which says that no company should profit at the expense of the others. Let $\left(R_{i}\right)$ be defined by (1.2). Under some regularity assumptions like the boundedness of $\left(X_{i}\right)$, Bühlmann (1984) proved that there exists a sequence $\left(k_{i}\right)$ such that $\mathbb{E}\left(R_{i} \Psi\right)=\mathbb{E}\left(X_{i} \Psi\right)$ for all $i$. Herein $\Psi$ is a positive random variable such that $\mathbb{E} \Psi=1$. The principle $P=$ $\mathbb{E}\left(X_{i} \Psi\right)$ is called the economic principle and $\Psi$ is said the price density. Bühlmann and Jewell (1979) also stated that under some mild assumptions there exists a sequence $\left(k_{i}\right)$ such that $\mathbb{E}\left(R_{i} \Psi\right)=\mathbb{E}\left(X_{i} \Psi\right)$ for all $i$, where $\left(R_{i}\right)$ is given by (1.6) and $\Psi$ is a price density (see also Lienhard, 1986, Aase, 1993). Other propositions based on ideas from the game theory have also appeared in actuarial literature. For $n=2$ Borch suggested to use the Nash solution of the bargain problem (see also Lemaire and Quairiere, 1986). The Kalai-Smorodinski solution is described in Lemaire (1991) along with a comprehensive review of other methods.

The aim of the paper is to provide an extension of both the Gerber condition and the Bühlmann-Jewell condition, see Section 2. In Section 3 one can find some applications of the result of Section 2. All the results presented in the paper are derived from elementary properties of convex functions and the Brouwer fixed point theorem. 


\section{MAIN RESULT}

Let $X_{i}$ denote a set of random variables defined on $(\Omega, \mathcal{S}, \mathbb{P})$ for $i=1,2, \ldots, n$. Let $\Pi$ be a mapping from $X=X_{i} \times \ldots \times X_{n}$ into the real numbers. The mapping $\Pi$ is called a $C$-functional at $\left(X_{i}\right)$ if there exists a random vector on $\Omega$, say $\left(\Pi_{1}^{\prime}, \ldots, \Pi_{n}^{\prime}\right)=\left(\Pi_{1,\left(X_{i}\right)}^{\prime}, \ldots, \Pi_{n,\left(X_{i}\right)}^{\prime}\right)$, such that for all $\left(Y_{i}\right) \in \mathcal{X}$

$$
\Pi\left(Y_{1}, \ldots, Y_{n}\right) \leq \Pi\left(X_{1}, \ldots, X_{n}\right)+\sum_{i=1}^{n} \mathbb{E}\left(\Pi_{i}^{\prime} \cdot\left(Y_{i}-X_{i}\right)\right) .
$$

The random variable $\Pi_{1}^{\prime}$ will be called an $i$ th partial subderivative of $\Pi$ at $\left(X_{j}\right)$. Any functional $\Pi$ satisfying (2.1) for each $\left(X_{i}\right) \in X$ will be called a $C$-functional.

\section{Examples:}

2.1. Letting $\pi_{i}\left(X_{i}\right)$ be a concave and Gâteaux differentiable functional, the mapping $\Pi=\sum_{i=1}^{n} \pi_{i}\left(X_{i}\right)$ is a C-functional and $\Pi_{1}^{\prime}=\pi_{i}^{\prime}\left(X_{i}\right)$, where $\pi_{i}^{\prime}$ means the Gâteaux derivative of $\pi_{i}$ (see Deprez and Gerber, 1999).

2.2. Suppose $w$ is a concave and nondifferentiable function on $\mathbb{R}$. Let $w_{-}^{\prime}$ and $w_{+}^{\prime}$ stand for the left derivative and the right derivative of $w$, respectively. Both derivatives exist at any point from the interior of the domain of $w$ and $\mathbb{E} w(Y)$ $\leq \mathbb{E} w(X)+\mathbb{E}\left[w_{X}^{\prime} \cdot(Y-X)\right]$ for each random variable $w_{X}^{\prime}$ such that

$$
w_{+}^{\prime}(X(\omega)) \leq w_{X}^{\prime}(\omega) \leq w_{-}^{\prime}(X(\omega)), \omega \in \Omega
$$

(see e.g. Rockafellar, 1970). Although the mapping $\Pi=\sum_{i=1}^{n} \mathbb{E} w\left(X_{i}\right)$ is not Gâteaux differentiable, it is a C-functional and $\Pi_{i}^{\prime}(\omega)=w_{X_{i}}^{\prime}(\omega), \omega \in \Omega$.

2.3. Suppose $\Pi$ attains its maximum at $\left(X_{j}^{*}\right)$. Then $\Pi$ is a $\mathrm{C}$-functional at $\left(X_{j}^{*}\right)$ and $\Pi_{i}^{\prime}=0$ for all $i$.

Remark 2.1. If $\Pi$ is a $\mathrm{C}$-functional then $\Pi$ is a concave mapping, i.e. $\alpha \Pi(X)+$ $(1-\alpha) \Pi(Y) \leq \Pi(\alpha X+(1-\alpha) Y)$ for all $0<\alpha<1$, where $X=\left(X_{j}\right)$ and $Y=\left(Y_{j}\right)$. This follows directly from the inequalities:

$$
\begin{aligned}
& \Pi(X) \leq \Pi(\alpha X+(1-\alpha) Y)+(1-\alpha) \sum_{i=1}^{n} \mathbb{E}\left(\Pi_{i}^{\prime} \cdot\left(X_{i}-Y_{i}\right)\right), \\
& \Pi(Y) \leq \Pi(\alpha X+(1-\alpha) Y)+\alpha \sum_{i=1}^{n} \mathbb{E}\left(\Pi_{i}^{\prime} \cdot\left(Y_{i}-X_{i}\right)\right),
\end{aligned}
$$

in which $\Pi_{i}^{\prime}=\Pi_{i, \alpha X+(1-\alpha) Y}^{\prime}$.

Given a functional $\Pi$, we find a solution of the constrained optimization problem:

$$
\max \Pi\left(R_{1}, \ldots, R_{n}\right) \text { s.t. }\left(R_{i}\right) \in \mathcal{R}_{L}^{U}(H)
$$


with

$$
R_{L}^{U}(H)=\left\{\left(R_{i}\right) ; \sum_{i=1}^{n} R_{i}=X, P_{i} \leq H_{i}\left(R_{i}\right), L_{i} \leq R_{i} \leq U_{i} \text { for } i \leq n-1\right\},
$$

where $H_{i}$ is a real-valued functional on $X_{i},-\infty<L_{i} \leq U_{i}<\infty$ are given random variables, and $\left(P_{i}\right)$ are fixed reals. Throughout the paper, $1_{A}$ denotes the indicator function of $A$.

\section{Theorem 1.}

(a) Assume there exist a rule $\left(R_{i}\right) \in R_{L}^{U}(H)$ and nonnegative reals $c_{1}, \ldots, c_{n-1}$ such that $\Pi$ and $\left(H_{i}\right)$ are $C$-functionals at $\left(R_{i}\right)$ and for $i \leq n-1$ :

(i) $\left(\Pi_{n}^{\prime}-\Pi_{i}^{\prime}-c_{i} H_{i}^{\prime}\right)_{+}\left(R_{i}-L_{i}\right)=0$,

(ii) $\left(c_{i} H_{i}^{\prime}-\Pi_{n}^{\prime}+\Pi_{i}^{\prime}\right)_{+}\left(U_{i}-R_{i}\right)=0$,

(iii) $H_{i}\left(R_{i}\right)=P_{i}$,

(iv) $\max _{1 \leq i \leq n} \mathbb{E}\left(\left(1+\left|\Pi_{i}^{\prime}\right|+\left|H_{i}^{\prime}\right|\right)\left|Y_{i}\right|\right)<\infty$ for $Y_{i}=L_{i}, U_{i}$, where $\Pi_{i}^{\prime}=\Pi_{i,\left(R_{j}\right)}^{\prime}$ and $H_{i}^{\prime}=H_{i, R_{i}}^{\prime}$. Then $\left(R_{i}\right)$ is a solution of the problem (2.2).

(b) Suppose $\Pi$ and $\left(H_{i}\right)$ are concave and Gâteaux differentiable. Suppose $\left(R_{i}\right)$ is a solution of (2.2) satisfying conditions (iii) and (iv) and suppose also that one of the following two assumptions holds:

A1. $H_{i}\left(R_{i}\right) \leq H_{i}\left(R_{i}+\left(U_{i}-R_{i}\right) 1_{A}+\left(L_{i}-R_{i}\right) 1_{B}\right)$ for $i \leq n-1$, where $A=\left\{c_{i} H_{i}^{\prime}-\right.$ $\left.\Pi_{n}^{\prime}+\Pi_{i}^{\prime}>0\right\}$ and $B=\left\{c_{i} H_{i}^{\prime}-\Pi_{n}^{\prime}+\Pi_{i}^{\prime}<0\right\}$ with $c_{i} \geq 0$,

A2. $H_{i}\left(R_{i}\right)<H_{i}\left(R_{i}+\left(U_{i}-R_{i}\right) 1_{C}\right)$, where $C=\left\{\Pi_{n}^{\prime}-\Pi_{i}^{\prime}-c_{i} H_{i}^{\prime} \leq 0\right\}$ with $c_{i} \geq 0$ and $H_{i}$ is increasing and continuous functional for $i \leq n-1$, i.e. $H_{i}(X) \leq$ $H_{i}(Y)$ if $X \leq Y$ and $H_{i}\left(X_{k}\right) \rightarrow H_{i}(X)$ for $X_{k} \uparrow X$ as $k \rightarrow \infty$, where $X_{k} \geq L_{i}$.

Then conditions ( $i)$-(ii) hold.

Proof. (a) Condition (iv) implies that $\mathbb{E} \hat{R}_{i}, \mathbb{E}\left(\Pi_{i}^{\prime} \hat{R}_{i}\right)$, and $\mathbb{E}\left(H_{i}^{\prime} \hat{R}_{i}\right)$ are finite for every $\left(\hat{R}_{i}\right) \in \mathcal{R}=\mathcal{R}_{L}^{U}(H)$. By the clearing condition, $\hat{R}_{n}-R_{n}=\sum_{i=1}^{n-1}\left(R_{i}-\hat{R}_{i}\right)$. From (2.1) with $Y_{i}=\hat{R}_{i}$ and $X_{i}=R_{i}$ it follows that for every $\left(\hat{R}_{i}\right) \in \mathcal{R}$

$$
\begin{aligned}
\Pi\left(\hat{R}_{1}, \ldots, \hat{R}_{n}\right) & \leq \Pi\left(R_{1}, \ldots, R_{n}\right)+\mathbb{E}\left(\sum_{i=1}^{n} \Pi_{i}^{\prime}\left(R_{i}-R_{i}\right)\right) \\
& \leq \Pi\left(R_{1}, \ldots, R_{n}\right)+\mathbb{E}\left(\sum_{i=1}^{n-1}\left(\Pi_{n}^{\prime}-\Pi_{i}^{\prime}\right)\left(R_{i}-\hat{R}_{i}\right)\right)
\end{aligned}
$$

with equality if $\left(\hat{R}_{i}\right)=\left(R_{i}\right)$. Write $Z_{i}=\Pi_{n}^{\prime}-\Pi_{i}^{\prime}$ for short. From the identity $x=$ $x_{+}-(-x)_{+}$, we get

$$
\mathbb{E}\left(Z_{i} \hat{R}_{i}\right)=\mathbb{E}\left[\left(Z_{i}-c_{i} H_{i}^{\prime}\right)_{+} \hat{R}_{i}\right]-\mathbb{E}\left[\left(c_{i} H_{i}^{\prime}-Z_{i}\right)_{+} \hat{R}_{i}\right]+c_{i} \mathbb{E}\left(H_{i}^{\prime} \hat{R}_{i}\right) .
$$


Since $L_{i} \leq \hat{R}_{i} \leq U_{i}$, we have

$$
\mathbb{E}\left(Z_{i} \hat{R}_{i}\right) \geq \mathbb{E}\left[\left(Z_{i}-c_{i} H_{i}^{\prime}\right)_{+} L_{i}\right]-\mathbb{E}\left[\left(c_{i} H_{i}^{\prime}-Z_{i}\right)_{+} U_{i}\right]+c_{i} \mathbb{E}\left(H_{i}^{\prime} \hat{R}_{i}\right)
$$

for every $\left(\hat{R}_{i}\right) \in \mathcal{R}$. From conditions (i) and (ii) it follows that equality occurs in (2.5) if $\hat{R}_{i}=R_{i}$. As a consequence of concavity of $H_{i}$ and condition (iii), we obtain

$$
\begin{aligned}
\mathbb{E}\left(H_{i}^{\prime} \hat{R}_{i}\right) \geq & \mathbb{E}\left(H_{i}^{\prime} R_{i}\right)+H_{i}\left(\hat{R}_{i}\right)-H_{i}\left(R_{i}\right)= \\
& \mathbb{E}\left(H_{i}^{\prime} R_{i}\right)+H_{i}\left(\hat{R}_{i}\right)-P_{i} \geq \mathbb{E}\left(H_{i}^{\prime} R_{i}\right)
\end{aligned}
$$

for every $\left(\hat{R}_{i}\right) \in \mathcal{R}$. Combining (2.4), (2.5), and (2.6) yields

$$
\begin{aligned}
& \Pi\left(\hat{R}_{1}, \ldots, \hat{R}_{n}\right) \leq \Pi\left(R_{1}, \ldots, R_{n}\right)+\sum_{i=1}^{n-1} \mathbb{E}\left(Z_{i} R_{i}\right)+ \\
& -\sum_{i=1}^{n-1}\left(\mathbb{E}\left[\left(Z_{i}-c_{i} H_{i}^{\prime}\right)_{+} L_{i}\right]-\mathbb{E}\left[\left(c_{i} H_{i}^{\prime}-Z_{i}\right)_{+} U_{i}\right]+c_{i} \mathbb{E}\left(H_{i}^{\prime} R_{i}\right)\right)
\end{aligned}
$$

for every $\left(\hat{R}_{i}\right) \in \mathcal{R}$. Equality in $(2.7)$ holds for $\left(\hat{R}_{i}\right)=\left(R_{i}\right)$ so

$$
\sum_{i=1}^{n-1}\left(\mathbb{E}\left(Z_{i} R_{i}\right)-\mathbb{E}\left[\left(Z_{i}-c_{i} H_{i}^{\prime}\right)_{+} L_{i}\right]+\mathbb{E}\left[\left(c_{i} H_{i}^{\prime}-Z_{i}\right)_{+} U_{i}\right]-c_{i} \mathbb{E}\left(H_{i}^{\prime} R_{i}\right)\right)=0 .
$$

As a consequence of $(2.7)$ and $(2.8), \Pi\left(\hat{R}_{1}, \ldots, \hat{R}_{n}\right) \leq \Pi\left(R_{1}, \ldots, R_{n}\right)$ for every $\left(\hat{R}_{i}\right) \in \mathcal{R}$, which completes the proof of part (a) of Theorem 1 .

(b) Let $\left(R_{i}\right)$ be a solution of the problem (2.2) satisfying conditions (iii) and (iv). Fix any $i, i \leq n-1$, and define $\left(R_{j}^{(t)}\right)$ as follows:

$$
R_{j}^{(t)}=R_{j} \text { for } j \neq i, n, \quad R_{i}^{(t)}=(1-t) R_{i}+t \hat{R}_{i}, \quad R_{n}^{(t)}=R_{n}-t\left(\hat{R}_{i}-R_{i}\right),
$$

where $t \in(0,1)$ and $\left(\hat{R}_{j}\right) \in \mathcal{R}$. Clearly, $\left(R_{j}^{(t)}\right) \in \mathcal{R}$ for each $t$. The function

$$
f(t)=\Pi\left(R_{1}^{(t)}, \ldots, R_{n}^{(t)}\right)+c_{i}\left(H_{i}\left(R_{i}^{(t)}\right)-P_{i}\right)
$$

has a maximum at $t=0$ and is concave. Hence $f_{+}^{\prime}(0) \leq 0$, where $f_{+}^{\prime}(0)$ denotes the right-hand derivative of $f$ at 0 . This implies

$$
\mathbb{E}\left(\left(\Pi_{n}^{\prime}-\Pi_{i}^{\prime}-c_{i} H_{i}^{\prime}\right)\left(R_{i}-\hat{R}_{i}\right)\right) \leq 0 .
$$

Let assumption A1 be fulfilled. Put

$$
\begin{aligned}
& \hat{R}_{j}=R_{j} \text { for } j \neq i, n, \\
& \hat{R}_{i}=R_{i}+\left(U_{i}-R_{i}\right) 1_{A}+\left(L_{i}-R_{i}\right) 1_{B}, \\
& \hat{R}_{n}=R_{n}-\left(\hat{R}_{i}-R_{i}\right),
\end{aligned}
$$


in which $A=\left\{c_{i} H_{i}^{\prime}-\Pi_{n}^{\prime}+\Pi_{i}^{\prime}>0\right\}$, and $B=\left\{c_{i} H_{i}^{\prime}-\Pi_{n}^{\prime}+\Pi_{i}^{\prime}<0\right\}$. Obviously, $\left(\hat{R}_{i}\right) \in \mathcal{R}$. From (2.9) we get

$$
\mathbb{E}\left[\left(\Pi_{n}^{\prime}-\Pi_{i}^{\prime}-c_{i} H_{i}^{\prime}\right)_{+}\left(R_{i}-L_{i}\right)\right]+\mathbb{E}\left[\left(c_{i} H_{i}^{\prime}-\Pi_{n}^{\prime}+\Pi_{i}^{\prime}\right)_{+}\left(U_{i}-R_{i}\right)\right] \leq 0 .
$$

This implies that conditions (i)-(ii) hold, which completes the proof under assumption A1. Let assumption A2 be fulfilled. Put

$$
\hat{R}_{j}=R_{j} \text { for } j \neq i, n, \quad \hat{R}_{i}=R_{i}+\left(U_{i}-R_{i}\right) 1_{C}, \quad \hat{R}_{n}=R_{n}-\left(U_{i}-R_{i}\right) 1_{C},
$$

where $C=\left\{\Pi_{n}^{\prime}-\Pi_{i}^{\prime}-c_{i} H_{i}^{\prime} \leq 0\right\}$. From (2.9) it follows that

$$
\mathbb{E}\left[\left(c_{i} H_{i}^{\prime}-\Pi_{n}^{\prime}+\Pi_{i}^{\prime}\right)_{+}\left(U_{i}-R_{i}\right)\right] \leq 0 .
$$

Since $\left(c_{i} H_{i}^{\prime}-\Pi_{n}^{\prime}+\Pi_{i}^{\prime}\right)_{+}\left(U_{i}-R_{i}\right) \geq 0$, condition (ii) holds. We now show that condition (i) is satisfied. Suppose it is untrue, that is, there exists $B_{0} \subset B=\left\{\Pi_{n}^{\prime}-\right.$ $\left.\Pi_{i}^{\prime}-c_{i} H_{i}^{\prime}>0\right\}$ such that $\mathbb{P}\left(B_{0}\right)>0$ and

$$
\left(\Pi_{n}^{\prime}-\Pi_{i}^{\prime}-c_{i} H_{i}^{\prime}\right)\left(R_{i}-L_{i}\right) 1_{B_{0}}>0 .
$$

Suppose also that $H_{i}\left(\hat{R}_{i}\right) \geq P_{i}$, where $\hat{R}_{i}=R_{i}+\left(U_{i}-R_{i}\right) 1_{C}+\left(L_{i}-R_{i}\right) 1_{B_{0}}$. The existence of $B_{0}$ follows from assumption A2 since $\hat{R}_{i} \uparrow \mathrm{R}_{i}+\left(U_{i}-R_{i}\right) 1_{C}$ as $B_{0}$ tends monotonically to the empty set. By (2.9)

$$
\mathbb{E}\left[\left(\Pi_{n}^{\prime}-\Pi_{i}^{\prime}-c_{i} H_{i}^{\prime}\right)\left(R_{i}-L_{i}\right) 1_{B_{0}}\right]+\mathbb{E}\left[\left(c_{i} H_{i}^{\prime}-\Pi_{n}^{\prime}+\Pi_{i}^{\prime}\right)_{+}\left(U_{i}-R_{i}\right)\right] \leq 0 .
$$

Hence $\left(\Pi_{n}^{\prime}-\Pi_{i}^{\prime}-c_{i} H_{i}^{\prime}\right)\left(R_{i}-L_{i}\right) 1_{B_{0}}=0$, which leads to a contradiction with (2.11). The proof is complete.

Remark 2.2. Theorem 1 (a) still holds with the problem (2.2) replaced by the following one:

$$
\max \Pi\left(R_{1}, \ldots, R_{n}\right) \text { s.t. }\left(R_{i}\right) \in \mathcal{R}_{L}^{U}\left(H_{=}\right),
$$

where

$$
\mathcal{R}_{L}^{U}\left(H_{=}\right)=\left\{\left(R_{i}\right) ; \sum_{i=1}^{n} R_{i}=X, P_{i}=H_{i}\left(R_{i}\right), L_{i} \leq R_{i} \leq U_{i} \text { for } i \leq n-1\right\} .
$$

Remark 2.3. From conditions (i)-(ii) of Theorem 1 we obtain that if $R_{i}>L_{i}$ then $\Pi_{n}^{\prime}-\Pi_{i}^{\prime} \leq c_{i} H_{i}^{\prime}$ and if $R_{i}<U_{i}$ then $\Pi_{n}^{\prime}-\Pi_{i}^{\prime} \geq c_{i} H_{i}^{\prime}$.

Hence conditions (i) and (ii) of Theorem 1 are equivalent to the following ones: 
$\Pi_{n}^{\prime}-\Pi_{i}^{\prime}=c_{i} H_{i}^{\prime}$ if $L_{i}<R_{i}<U_{i}$

$\Pi_{n}^{\prime}-\Pi_{i}^{\prime} \geq c_{i} H_{i}^{\prime}$ if $R_{i}=L_{i}$,

$\Pi_{n}^{\prime}-\Pi_{i}^{\prime} \leq c_{i} H_{i}^{\prime}$ if $R_{i}=U_{i}$,

where $i=1,2, \ldots, n-1$. Clearly, for $\Pi=\sum_{i=1}^{n} k_{i} \mathbb{E} u_{i}\left(R_{i}\right)$ and $H_{i}\left(R_{i}\right)=P_{i}=0,1 \leq$ $i \leq n$, we obtain the Bühlmann-Jewell condition with $\Lambda=k_{n} u_{n}^{\prime}\left(R_{n}\right)$.

In a similar manner as in the proof of Theorem 1 we may easily show that conditions (i)-(ii) of Theorem 1 have to be replaced by:

- $\left(c_{i} H_{i}^{\prime}-\Pi_{n}^{\prime}+\Pi_{i}^{\prime}\right)_{+}\left(U_{i}-R_{i}\right)=0$, $\left(\Pi_{n}^{\prime}-\Pi_{i}^{\prime}-c_{i} H_{i}^{\prime}\right)_{+}=0, i=1, \ldots, n-1$,

provided $R_{i} \leq U_{i}$ for all $R_{i}$, that is, if the problem (2.2) with $\left(L_{i}\right)=(-\infty)$ is considered.

- $\left(c_{i} H_{i}^{\prime}-\Pi_{n}^{\prime}+\Pi_{i}^{\prime}\right)_{+}=0$, $\left(\Pi_{n}^{\prime}-\Pi_{i}^{\prime}-c_{i} H_{i}^{\prime}\right)_{+}\left(R_{i}-L_{i}\right)=0, i=1, \ldots, n-1$,

provided $\left(U_{i}\right)=(\infty)$. This extends the Gerber condition. In fact, putting $H_{i}=$ $P_{i}=0$ and $\Pi=\sum_{i=1}^{n} k_{i} u_{i}\left(R_{i}\right)$ we get the condition: if $k_{i} u_{i}^{\prime}\left(R_{i}\right)<\max \left\{k_{j} u_{j}^{\prime}\left(R_{j}\right)\right.$; $1 \leq j \leq n\}$ then $R_{i}=L_{i}$.

- $c_{i} H_{i}^{\prime}=\Pi_{n}^{\prime}-\Pi_{i}^{\prime}, i=1, \ldots, n-1$,

provided $\left(L_{i}\right)=(-\infty)$ and $\left(U_{i}\right)=(\infty)$. For $H_{i}=P_{i}=0$ we obtain the extended Borch condition.

\section{Applications}

\subsection{Extension of Bühlmann result}

As mentioned in Section 1 of the paper, Bühlmann (1984) proved that if the sequence $\left(X_{i}\right)$ is assumed to be bounded and if the Lipschitz condition is imposed on the Arrow-Pratt risk aversion function $\rho_{i}$ defined by $\rho_{i}=-u_{i}^{\prime \prime} / u_{i}^{\prime}$, then there exists a solution of $(1.1)$, say $\left(R_{i}\right)$, which satisfies the nonprofit condition with the economic principle. We now extend this result to cover the case of other premium principles. We first prove the result of Bühlmann for economic principle without restrictive assumptions on $\left(X_{i}\right)$ and $\rho_{i}$.

\section{Theorem 2.}

Assume all $u_{i}$ are differentiable and strictly concave on $\mathbb{R}$, assume $u_{i}^{\prime}(\mathbb{R})=(0, \infty)$ for all $i$, and assume $\max _{1 \leq i \leq n} \mathbb{E}\left|X_{i} \Psi\right|<\infty$. Then there are coefficients $\left(k_{i}\right)$ such that $\mathbb{E}\left(R_{i} \Psi\right)=\mathbb{E}\left(X_{i} \Psi\right)$ for $i=1,2, \ldots, n$, where $\left(R_{i}\right)$ is a solution of $(1.1)$.

Proof. Observe that it is enough to prove that $\mathbb{E}\left(R_{i} \Psi\right)=\mathbb{E}\left(X_{i} \Psi\right)$ for $i \leq n-1$ because of the clearing condition. Define the map $F=\left(F_{1}, \ldots, F_{n-1}\right)$ from $\mathbb{R}^{n-1}$ 
to $\mathbb{R}^{n-1}$ as follows: $F_{i}\left(x_{i}\right)=\mathbb{E}\left(X_{i} \Psi\right)-\mathbb{E}\left(R_{i} \Psi\right)+x_{i}$, where $R_{i}$ is the solution of the problem (1.1) such that $R_{i}(0)=x_{i}$ (cf. Bühlmann, 1984). Clearly,

$$
R_{i}(x)=u_{i}^{*}\left(\frac{u_{i}^{\prime}\left(x_{i}\right)}{u_{n}^{\prime}\left(x_{n}\right)} u_{n}^{\prime}\left(h^{*}(x)\right)\right), i=1, \ldots, n, x \in \mathbb{R},
$$

where $x_{n}=-\sum_{i=1}^{n-1} x_{i}$ and

$$
h(x)=\sum_{i=1}^{n} u_{i}^{*}\left(\frac{u_{i}^{\prime}\left(x_{i}\right)}{u_{n}^{\prime}\left(x_{n}\right)} u_{n}^{\prime}(x)\right)
$$

We now show that any solution of (1.1) satisfies the Lipschitz condition with constant 1, i.e. $\left|R_{i}(x)-R_{i}(0)\right| \leq|x|$ for all $x$. Obviously, it is enough to show that both the functions $x \rightarrow R_{i}(x)$ and $x \rightarrow x-R_{i}(x)$ are increasing for each $i$. Recall that $u_{i}^{\prime}$ is decreasing. Since the composition of two decreasing function is increasing, $x \rightarrow R_{i}(x)$ is increasing. Since $\left(R_{j}\right)$ are increasing and $u_{i}^{\prime}$ is decreasing, we have

$$
u_{i}^{\prime}\left(x-R_{i}(x)\right)=u_{i}^{\prime}\left(\sum_{j \neq i} R_{j}(x)\right)>u_{i}^{\prime}\left(\sum_{j \neq i} R_{j}(y)\right)=u_{i}^{\prime}\left(y-R_{i}(y)\right)
$$

for $x<y$ so $x-R_{i}(x)<y-R_{i}(y)$ for $x<y$, as expected. By the dominated convergence theorem and continuity of the function $\left(x_{1}, \ldots, x_{n}\right) \rightarrow R_{i}(x)$ for any fixed $x$ and $i$, the map $F$ is continuous. From the Lipschitz condition we get that for every $x_{i} \in \mathbb{R}$

$$
\left|F_{i}\left(x_{i}\right)\right| \leq \mathbb{E}\left(\Psi\left|X_{i}-R_{i}(X)+R_{i}(0)\right|\right) \leq \mathbb{E}\left(\Psi\left(\left|X_{i}\right|+|X|\right)\right)<\infty,
$$

where $R_{i}$ is given by (3.1). Let $M=\left[-m_{1}, m_{1}\right] \times \ldots \times\left[-m_{n-1}, m_{n-1}\right]$ in which $m_{i}=$ $\mathbb{E}\left(\Psi\left(\left|X_{i}\right|+|\mathrm{X}|\right)\right)$. Let $\left.F\right|_{M}$ denote the restriction of $F$ to $M$. Since $\left.F\right|_{M}: M \rightarrow M$ is a continuous function defined on a compact and convex set, the Brouwer theorem implies that there is a fixed point, say $\left(x_{i}^{*}\right)$, of mapping $\left.F\right|_{M}$, that is, $\mathbb{E}\left(R_{i} \Psi\right)=\mathbb{E}\left(X_{i} \Psi\right)$ for each $i$, where $R_{i}(0)=x_{i}^{*}$. Since $u_{i}^{\prime}>0$, it is clear that $k_{i}=$ $k_{n} u_{i}^{\prime}\left(x_{i}^{*}\right) / u_{n}^{\prime}\left(x_{n}^{*}\right)>0$ for $i \leq n-1$, as desired.

Remark 3.1. Theorem 2 also holds for other types of utility function. For instance, if $u_{i}(x)=\ln x$ for all $i$, then a solution of (1.1) satisfying the nonprofit condition is given by

$$
R_{i}(X)=\frac{\mathbb{E}\left(X_{i} \Psi\right)}{\mathbb{E}(X \Psi)} X, \quad i=1,2, \ldots, n .
$$

We now provide a Bühlmann type result for premiums of the form $H_{i}(X)=$ $\mathbb{E} X+h_{i}(\mathbb{D} X)$, where $h_{i}$ is a nondecreasing differentiable and convex function 
defined on $[0, \infty)$ and $h_{i}(0)=0$. Examples include the standard deviation principle, the variance principle, and the mixed principle (cf. Section 3.5 of the paper).

\section{Theorem 3.}

Suppose $0<\mathbb{D} X<\infty$. Under the assumptions of Theorem 2 there exist coefficients $\left(k_{i}\right)$ such that the solution of $(1.1)$, say $\left(R_{i}\right)$, satisfies the condition $H_{i}\left(R_{i}\right)=H_{i}\left(X_{i}\right)$ for $i=1,2, \ldots, n-1$, with $H_{i}\left(R_{i}\right)=\mathbb{E} R_{i}+h_{i}\left(\mathbb{D} R_{i}\right)$.

Proof. Let $F_{i}\left(x_{i}\right)=H_{i}\left(X_{i}\right)-H_{i}\left(R_{i}-x_{i}\right)$ define the map $F=\left(F_{1}, \ldots, F_{n-1}\right)$ from $\mathbb{R}^{n-1}$ to $\mathbb{R}^{n-1}$, where $R_{i}(0)=x_{i}$ for each $i$. It is easy to check that the principle $H_{i}(X)=$ $-\mathbb{E} X-h_{i}(\mathbb{D} X)$ with a differentiable and convex function $h_{i}$ is a C-functional and $H_{i, X}^{\prime}=-1-h_{i}^{\prime}(\mathbb{D} X)(X-\mathbb{E} X) / \mathbb{D} X$ for $\mathbb{D} X>0$ and $H_{i, X}^{\prime}=-1$ otherwise. Therefore

$$
\begin{aligned}
\mathbb{E}\left(H_{i, X_{i}}^{\prime}\left(X_{i}-R_{i}+x_{i}\right)\right) & \leq H_{i}\left(X_{i}\right)-H_{i}\left(R_{i}-x_{i}\right) \leq \\
& \leq \mathbb{E}\left(H_{i}^{\prime}\left(X_{i}-R_{i}+x_{i}\right)\right)
\end{aligned}
$$

(see Deprez and Gerber, 1985, Theorem 8). Herein $H_{i}^{\prime}=H_{i, R_{i}}^{\prime}$. Hence for every $x_{i} \in \mathbb{R}$

$$
\begin{array}{r}
\left|\left(H_{i}\left(X_{i}\right)-H_{i}\left(R_{i}-x_{i}\right)\right)\right| \leq \max \left\{\left|\mathbb{E}\left(H_{i, X_{i}}^{\prime}\left(X_{i}-R_{i}+x_{i}\right)\right)\right|,\right. \\
\left.\left|\mathbb{E}\left(H_{i}^{\prime}\left(X_{i}-R_{i}+x_{i}\right)\right)\right|\right\} .
\end{array}
$$

Recall that $\left|R_{i}-x_{i}\right|=\left|R_{i}(X)-R_{i}(0)\right| \leq|X|$ (see the proof of Theorem 2). By the Lipschitz condition

$$
\begin{aligned}
\left|\mathbb{E}\left(H_{i, X_{i}}^{\prime}\left(X_{i}-R_{i}+x_{i}\right)\right)\right| & \leq \mathbb{E}\left(\left|H_{i, X_{i}^{\prime}}\right|\left(\left|X_{i}\right|+\left|R_{i}-x_{i}\right|\right)\right. \\
& \leq \mathbb{E}\left(\left|H_{i, X_{i}}^{\prime}\right|\left(\left|X_{i}\right|+|X|\right)<\infty\right.
\end{aligned}
$$

for every solution of (1.1) such that $R_{i}(0)=x_{i}$. Furthermore, if $\mathbb{D} R_{i}=0$ then by the Lipschitz condition

$$
\left|\mathbb{E}\left[H_{i}^{\prime}\left(X_{i}-R_{i}+x_{i}\right)\right]\right| \leq \mathbb{E}\left(\left|X_{i}\right|+|X|\right)<\infty .
$$

If $\mathbb{D} R_{i}>0$ then

$$
\begin{aligned}
& \left|\mathbb{E}\left[H_{i}^{\prime}\left(X_{i}-R_{i}+x_{i}\right)\right]\right| \leq \mathbb{E}\left(\left|X_{i}\right|+|X|\right)+ \\
& +\frac{h^{\prime}\left(\mathbb{D} R_{i}\right)}{\mathbb{D} R_{i}} \mid \mathbb{E}\left[\left(R_{i}-\mathbb{E} R_{i}\right)\left(X_{i}-R_{i}+x_{i}\right)\right] .
\end{aligned}
$$

From the Cauchy-Schwarz inequality, the inequality $(x+y)^{2} \leq 2\left(x^{2}+y^{2}\right)$, and the Lipschitz condition it follows

$$
\begin{aligned}
\left(\mathbb{E}\left[\left(R_{i}-\mathbb{E} R_{i}\right)\left(X_{i}-R_{i}+x_{i}\right)\right]\right)^{2} & \leq \mathbb{E}\left(X_{i}-R_{i}+x_{i}\right)^{2} \mathbb{D}^{2} R_{i} \\
& \leq 2\left(\mathbb{E} X_{i}^{2}+\mathbb{E} X^{2}\right) \mathbb{D}^{2} R_{i} .
\end{aligned}
$$


By the Lipschitz condition

$$
\mathbb{D}^{2} R_{i}=\mathbb{D}^{2}\left(R_{i}-x_{i}\right) \leq \mathbb{E}\left(R_{i}-x_{i}\right)^{2} \leq \mathbb{E} X^{2} .
$$

Combining (3.6)-(3.8) with the assumption that $h^{\prime}$ is nondecreasing yields

$$
\begin{aligned}
& \left|\mathbb{E}\left(H_{i}^{\prime}\left(X_{i}-R_{i}+x_{i}\right)\right)\right| \leq \mathbb{E}\left(\left|X_{i}\right|+|X|\right)+ \\
& \quad+h^{\prime}\left(\mathbb{E} X^{2}\right)\left(2\left[\mathbb{E} X_{i}^{2}+\mathbb{E} X^{2}\right]\right)^{1 / 2}<\infty .
\end{aligned}
$$

From (3.3)-(3.5) and (3.9) it follows that for every $\left(R_{i}\right)$ satisfying (1.1) with $R_{i}(0)=x_{i}$

$$
\left|H_{i}\left(X_{i}\right)-H_{i}\left(R_{i}-x_{i}\right)\right| \leq m_{i}
$$

with some reals $m_{i}$. Moreover, $H_{i}$ is continuous, i.e. $H_{i}\left(R_{n}\right) \rightarrow H_{i}(R)$ if $R_{n} \rightarrow R$ a.s. and $\left|R_{n}\right| \leq Y$ for all $n$ with $H_{i}(Y)<\infty$. Consequently, $\left.F\right|_{M}$ is a continuous function from $M=\left[-m_{1}, m_{1}\right] \times \ldots \times\left[-m_{n-1}, m_{n-1}\right]$ to $M$. By the Brouwer theorem, there is a fixed point, say $\left(x_{i}^{*}\right)$, of $\left.F\right|_{M}$, that is, $H\left(X_{i}\right)=H\left(R_{i}\right)$ for each $i \leq$ $n-1$, where $R_{i}(0)=x_{i}^{*}$, as desired.

Theorem 3 also applies to the following premium calculation principles:

- exponential principle

$$
\begin{array}{ll}
\text { - } \text { exponential principle } & H(R)=(1 / \beta) \log \mathbb{E} e^{\beta R}, \\
\text { - } \text { semi-variance principle } & H(R)=\mathbb{E} R+\beta \mathbb{E}(R-\mathbb{E} R)_{+}^{2}, \\
\text { - } \text { semi-deviation principle } & P=\mathbb{E} R+\beta\left(\mathbb{E}(R-\mathbb{E} R)_{+}^{2}\right)^{1 / 2}, \\
\text { - } \text { covariance principle } & P=\mathbb{E} R+2 \beta \mathbb{D}^{2} R-\beta \operatorname{Cov}(R, Y \\
\text { - } \text { Dutch principle } & P=\mathbb{E} R+\beta \mathbb{E}(R-\mathbb{E} R)_{+},
\end{array}
$$$$
\text { - covariance principle } \quad P=\mathbb{E} R+2 \beta \mathbb{D}^{2} R-\beta \operatorname{Cov}(R, Y) \text {, }
$$

- Dutch principle

among others. For instance, if the $i$ th insurer uses the exponential principle with the index $\beta_{i}$ then it is enough to show that there is a sharing rule, say $\left(R_{i}\right)$, such that $\mathbb{E} \exp \left(\beta_{i} R_{i}\right)=\mathbb{E} \exp \left(\beta_{i} X_{i}\right)$ for $i \leq n-1$. Putting $H_{i}(R)=-\mathbb{E} \exp \left(\beta_{i} R\right)$, we get $H_{i, R}^{\prime}=-\beta_{i} \exp \left(\beta_{i} R\right)$. Following similar lines as in the proof of Theorem 3 we obtain that $\left|H_{i}\left(X_{i}\right)-H_{i}\left(R_{i}-x_{i}\right)\right|$ is bounded in $x_{i} \in \mathbb{R}$ if $\mathbb{E} \exp \left(\beta_{i} X\right)<\infty$. The rest of the proof is straightforward.

It is worth to emphasize that the rule (1.2) may not satisfy the nonprofit condition for $i=n$.

\section{Examples}

3.1. Let $u_{i}(x)=\ln x$ for all $i$ and let $H_{i}(R)=\mathbb{E} R+\beta_{i} \mathbb{D} R$. It is easy to check that the solution of (1.1) is the rule $R_{i}=\left(k_{i} / \sum_{j=1}^{n} k_{j}\right) X$ and there exists a rule satisfying nonprofit condition for all $i$ if and only if

$$
\sum_{i=1}^{n} \frac{\mathbb{E} X_{i}+\beta_{i} \mathbb{D} X_{i}}{\mathbb{E} X+\beta_{i} \mathbb{D} X}=1
$$

If $\left(X_{i}\right)$ are independent random variables and if $\beta_{i}=\beta$ for all $i$, then the nonprofit condition does not hold for $i=n$ since $\mathbb{D}\left(X_{1}+\ldots+X_{n}\right)<\mathbb{D}\left(X_{1}\right)+\ldots+\mathbb{D}\left(X_{n}\right)$. 
3.2. Suppose $H_{i}(X)=H(X)$ for all $i$, where $H$ is an additive and comonotonic premium principle, that is,

i) $H\left(X_{1}+X_{2}\right)=H\left(X_{1}\right)+H\left(X_{2}\right)$ for independent risks $X_{i}$,

ii) $H\left(R_{1}\right)+H\left(R_{2}\right) \leq H\left(R_{1}+R_{2}\right)$ for nondecreasing functions $R_{i}=R_{i}(X)$.

An example is the exponential principle. Property (ii) follows from the wellknown inequality: $\mathbb{E} f(X) \mathbb{E} g(X) \leq \mathbb{E}(f(X) g(X))$ for all nondecreasing functions $f, g$ such that the expectations exist (see e.g. Rolski et al., 1999). Suppose Theorem 3 holds for $\left(H_{i}\right)$ with $H_{i}=H$ for all $i$ and suppose the risks $\left(X_{i}\right)$ are independent. Then there is a rule $\left(R_{i}\right)$ such that $H\left(R_{i}\right)=H\left(X_{i}\right)$ for $1 \leq i \leq n-1$ and

$$
\sum_{i=1}^{n} H\left(R_{i}\right) \leq H\left(\sum_{i=1}^{n} R_{i}\right)=H\left(\sum_{i=1}^{n} X_{i}\right)=\sum_{i=1}^{n} H\left(X_{i}\right)
$$

because $x \rightarrow R_{i}(X)$ are nondecreasing. This implies that $H\left(R_{n}\right) \leq H\left(X_{n}\right)$.

\subsection{Pool}

Consider $n$ insurance companies which would like to exchange reinsurance. Let $X_{i}$ stand for the aggregate claim amount of the $i$ th company. The premium of the company is calculated according to the expected value principle with the safety loading coefficient $\beta_{i}$, i.e. $P_{i}=\left(1+\beta_{i}\right) \mathbb{E} X_{i}$. The companies seek for a risk exchange $\left(R_{i}\right)$ such that the sum of variances of retained risks will be as small as possible provided the nonprofit condition is fulfilled, that is, $\mathrm{P}_{i}=\left(1+\beta_{i}\right) \mathbb{E} R_{i}$ for all $i$. Hence the following problem should be solved

$$
\min \sum_{i=1}^{n} \mathbb{D}^{2} R_{i} \text { s.t. } \sum_{i=1}^{n} R_{i}=X, P_{i}=\left(1+\beta_{i}\right) \mathbb{E} R_{i}, R_{i} \geq 0 \text { for } i \leq n .
$$

\section{Theorem 4.}

Suppose $\mathbb{E} X=\sum_{i=1}^{n} P_{i} /\left(1+\beta_{i}\right)$ with $X=\sum_{i=1}^{n} X_{i}$ and suppose $P_{i} /\left(1+\beta_{i}\right) \leq P_{j} /$ $\left(1+\beta_{j}\right)$ for $i<j$. Then a solution of the problem (3.11) is given by

$$
R_{i}^{*}(X)=\left(h^{*}(X)-b_{i}\right)_{+} \text {for } i=1,2, \ldots, n,
$$

where $h^{*}$ is the inverse function of $h(x)$ defined by $h(x)=\sum_{i=1}^{n}\left(x-b_{i}\right)_{+}$while $0=$ $b_{n} \leq b_{n-1} \leq \ldots \leq b_{1}$ are reals such that $\left(1+\beta_{i}\right) \mathbb{E} R_{i}^{*}=P_{i}$ for $1 \leq i \leq n$.

Proof. In Theorem 1 (a) we put $\Pi=-\sum_{i=1}^{n} \mathbb{D}^{2} R_{i}$ and $H_{i}=P_{i}-\left(1+\beta_{i}\right) \mathbb{E} R_{i}$ for $\mathrm{i}=1,2, \ldots, n$ (see Remark 2.2). The extended Gerber condition is as follows:

$$
\Pi_{n}^{\prime}-\Pi_{i}^{\prime}=c_{i} H_{i}^{\prime} \text { if } R_{i}>0 \text { and } \Pi_{n}^{\prime}-\Pi_{i}^{\prime} \geq c_{i} H_{i}^{\prime} \text { if } R_{i}=0 .
$$

Obviously, $\Pi_{i}^{\prime}=-2\left(R_{i}-\mathbb{E} R_{i}\right)$ and $H_{i}^{\prime}=-\left(1+\beta_{i}\right)$. This implies 
$R_{n}(x)-R_{i}(x)=d_{i}$ if $R_{i}(x)>0$,

$R_{n}(x)-R_{i}(x) \leq d_{i}$ if $R_{i}(x)=0$,

for $i=1,2, \ldots, n-1$, where $\left(d_{i}\right)$ are reals. The rule $\left(R_{j}^{*}\right)$ satisfies the extended Gerber condition. Since $b_{i} \leq b_{1}$ for all $i$ and $\mathbb{E} R_{1}^{*}(X)=n^{-1} \mathbb{E}\left(X-b_{1}\right)_{+}=P_{1} /\left(1+\beta_{1}\right)$, the proof of existence of reals $\left(b_{i}\right)$ such that $\left(1+\beta_{i}\right) \mathbb{E} R_{i}^{*}=P_{i}$ for all $i$ is similar to that of Theorem 3 with $b=\left(b_{1}, \ldots, b_{n-1}\right) \rightarrow\left(F_{1}(b), \ldots, F_{n-1}(b)\right)$, where $F_{i}(b)=$ $\left|\left(1+\beta_{i}\right) \mathbb{E} R_{i}^{*}-P_{i}\right|+b_{i} \geq 0$. We omit the details.

\subsection{Optimal insurance}

Suppose insurance is written on the aggregate loss, i.e. the insurer covers $R=$ $R(X)$ and the insured covers $X-R$, where $X$ means the global risk and $R$ means a random variable defined on $(\Omega, S, \mathbb{P})$, called the compensation rule. Assume the insurer uses the expected value principle of premium calculation with a safety loading coefficient $\beta>0$. Given an insurer's premium $P$, the policyholder has a wealth $m$ after paying for insurance and wants to have an arrangement which maximizes a utility function of his retained risk under a restriction on insurer's cover, namely, $L \leq R \leq U$, where $L, U$ are random variables such that $0 \leq L \leq U$. Hence the following problem arises

$$
\max \mathbb{E} u(m+R-X) \text { s.t. }(1+\beta) \mathbb{E} R=P, L \leq R \leq U,
$$

in which $u$ is a increasing differentiable and concave function. A solution of the problem (3.12) with $L=0$ and $U=X$ was given by Arrow (1963). Throughout the paper we use the following notation

$$
\langle a\rangle_{U L}=\min \{U, \max \{L, a\}\} .
$$

\section{Theorem 5.}

Suppose $\mathbb{E} L<P \leq \mathbb{E} U$. Then a solution of the problem (3.12) is the limited stop loss contract defined by $R^{*}=\langle X-d\rangle_{U L}$, where $d$ is a nonnegative real such that $\mathbb{E} R^{*}=P$.

Proof. See Pesonen (1984) or Daykin et al. (1994) for the proof via an extension of the Jensen inequality.

We now provide an extension of Theorem 5. Suppose the policyholder is willing to have a contract which maximizes his utility function, say $\mathbb{E} u(m+R-X)$, while the insurer wants to have a contract such that $\mathbb{E} v(M-R) \geq P$, where $v$ and $M$ means the insurer's utility function and the insurer's wealth, respectively, and $P$ is a fixed real. We assume that both the insured and the insurer are risk averse, i.e. $u$ and $v$ are differentiable increasing and concave functions. We also assume that $u$ is strictly concave. This leads to the following problem

$$
\max \mathbb{E} u(m+R-X) \text { s.t. } \mathbb{E} v(M-R) \geq P, L \leq R \leq U .
$$


Put $u(t, c)=t+u^{\prime-1}\left(c v^{\prime}(t)\right)$ for $c>0$. In order that $u(t, c)$ would be well-defined we assume that $v^{\prime}\left(D_{v}\right)=u^{\prime}\left(D_{u}\right)=(0, \infty)$. Let $x \rightarrow u^{*}(x, c)$ means the inverse function of $t \rightarrow u(t, c)$. Define

$$
R_{c}=\left\langle M-u^{*}(m+M-X, c)\right\rangle_{U L}
$$

\section{Theorem 6.}

Assume $\mathbb{E}\left(|X|+\left|X u^{\prime}(X)\right|+\left|X v^{\prime}(X)\right|\right)<\infty$ and assume $\mathbb{E} v\left(M-R_{0+}\right)<P<\mathbb{E} v(M-$ $\left.R_{\infty}\right)$. Then a solution of the problem (3.13) is the compensation rule $R_{c}$ defined by (3.14) with c being such that $\mathbb{E} v\left(M-R_{c}\right)=P$.

Proof. We apply Theorem 1 (a) with $n=2, R_{1}=R, R_{2}=X-R, \Pi=\mathbb{E} u\left(m-R_{2}\right)$, $\mathrm{H}_{1}=\mathbb{E} v\left(M-R_{1}\right), H_{2}=0, L_{1}=L$, and $U_{1}=U$. Clearly, $\Pi_{1}^{\prime}=0, \Pi_{2}^{\prime}=-u^{\prime}(m+R-X)$ and $H_{1}^{\prime}=-v^{\prime}(M-R)$. The Bühlmann-Jewell condition is as follows:

$c v^{\prime}(M-R)=u^{\prime}(m+R-X)$ if $L<R<U$

$c v^{\prime}(M-R) \geq u^{\prime}(m+R-X)$ if $R=L$,

$c v^{\prime}(M-R) \leq u^{\prime}(m+R-X)$ if $R=U$,

with $c>0$ (see Remark 2.3). The equation $c v^{\prime}(M-R)=u^{\prime}(m+R-X)$ is equivalent to $u(M-R, c)=m+M-X$. Hence the solution of $c v^{\prime}(M-R)=u^{\prime}(m+R-X)$ is given by $R=M-u^{*}(m+M-X, c)$. Define $R_{c}$ by (3.14). Clearly, $\left(R_{c}\right)$ satisfies the Bühlmann-Jewell condition. We now show the existence of a real $c$ such that $\mathbb{E} v\left(M-R_{c}\right)=P$. Put $h(c)=\mathbb{E} v\left(M-R_{c}\right)-P$, where $c>0$. By the dominated convergence theorem, $h$ is continuous. Moreover, $h(0+)<0$ and $h(\infty)>0$. Hence there is a real $c>0$ such that $\mathbb{E} v\left(M-R_{c}\right)=P$. The proof is complete.

Example 3.3. Suppose both the policyholder and the insurer use the exponential utility function with index $\alpha$ and $\beta$, respectively. A solution of the following problem:

$$
\max \mathbb{E} u_{\alpha}(m+R-X) \text { s.t. } \mathbb{E} u_{\beta}(M-R) \geq P, L \leq R \leq U,
$$

is given by

$$
R^{*}=\left\langle\frac{\alpha}{\alpha+\beta}(X-d)\right\rangle_{U L},
$$

provided $\mathbb{E} X<\infty$ and $1-\mathbb{E} e^{\beta(U-M)}<\beta P<1-\mathbb{E} e^{\beta(L-M)}$. Herein $d$ is a real such that $P=\mathbb{E} u_{\beta}\left(M-R^{*}\right)$.

Example 3.4. Let the wealth of the insurer be greater than or equal to the wealth of the policyholder, i.e. $m \leq M$. Suppose both the policyholder and the insurer use the logarithmic utility. Assume $X<m$ and assume $\mathbb{E} \log (M-U)<$ $P<\mathbb{E} \log (M-L)$. Then a solution of the problem

$$
\max \mathbb{E} \log (m+R-X) \text { s.t. } \mathbb{E} \log (M-R) \geq P, L \leq R \leq U
$$


is the rule

$$
R^{*}=\left\langle\frac{c}{1+c}\left(X-m+\frac{M}{c}\right)\right\rangle_{U L}
$$

in which $c>0$ is such that $\mathbb{E} \log \left(M-R^{*}\right)=P$.

Example 3.5. Given $0<\alpha<1$, let $u(x)=\frac{1}{1-\alpha}\left(x^{1-\alpha}-1\right), x>0$, be the common power utility function of the policyholder and the insurer. Assume that $X<m \leq M$. From Theorem 6 we get that if $\mathbb{E}\left((M-U)^{1-\alpha}\right)<(1-\alpha) P+1<\mathbb{E}\left((M-L)^{1-\alpha}\right)$, then a solution of the problem:

$$
\max \mathbb{E} u(m+R-X) \text { s.t. } \mathbb{E} u(M-R) \geq P, L \leq R \leq U,
$$

is the contract

$$
R^{*}=\left\langle\frac{c^{1 / \alpha}}{1+c^{1 / \alpha}}\left(X-m+\frac{M}{c^{1 / \alpha}}\right)\right\rangle_{U L}
$$

where $c$ is such that $\mathbb{E} u\left(M-R^{*}\right)=P$.

Remark 3.2. Observe that in Examples 3.3-3.5 the optimal contract is a limited combination of a stop loss and quota share.

\subsection{Dividends payments}

Suppose the insurer offers a profit sharing plan to the policyholder because of the threat of self-insurance on the part of the good risks. He will refund some part of the profit he makes on the policy. Let $R$ stand for the refund, let $P$ denote the insurer's premium, and let $X$ denote the total claim amount. Since the insurer refunds a part of the profit, one natural constraint on the set of all refunds is $0 \leq R \leq$ $(P-X)_{+}$. More restrictive constraints are also of great actuarial interest. We assume $L \leq R \leq U$ where $0 \leq L \leq U \leq(P-X)_{+}$. Denote by $u$ and $v$ utility function of the insured and the insurer, respectively. Adopt same assumptions for $u$ and $v$ and definition of $u^{*}$ as in Section 3.3. Given a real $q$, a solution of the following problem

$$
\max \mathbb{E} u(m-P+R) \text { s.t. } \mathbb{E} v(M-R-X+P) \geq q, L \leq R \leq U,
$$

is a Pareto-optimal dividends policy. The case when the insured is risk neutral ( $u$ is linear) was treated by Gerber and Jones (1974). The work of Gerber and Jones was extended by Vandebroek (1988) who assumed that the insured is riskaverse ( $u$ is concave). In both cited papers, $L=0$ and $U=(P-X)_{+}$. We provide a further extension.

\section{Theorem 7.}

Let $\mathbb{E}|X|, \mathbb{E}\left|X u^{\prime}(X)\right|$, and $\mathbb{E}\left|X v^{\prime}(X)\right|$ be finite. Then a solution of the problem (3.19) is given by 


$$
R_{c}=\left\langle M-X+P-u^{*}(m+M-X, c)\right\rangle_{U L},
$$

with $c$ being such that $\mathbb{E} v\left(M-R_{c}-X+P\right)=q$ provided $\mathbb{E} v\left(M-R_{0+}-X+P\right)<q<$ $\mathbb{E} v\left(M-R_{\infty}-X+P\right)$.

Proof. Put in Theorem 1 (a): $n=2, R_{1}=X-P+R, R_{2}=P-R, \Pi=\mathbb{E} u\left(m-R_{2}\right)$, $H_{1}=\mathbb{E} v\left(M-R_{1}\right), P_{1}=q, L_{1}=L+X-P$, and $U_{1}=U+X-P$. Clearly, $\Pi_{1}^{\prime}=0$, $\Pi_{2}^{\prime}=-u^{\prime}(m-P+R)$, and $H_{1}^{\prime}=-v^{\prime}(M-X+P-R)$. The rest of the proof is omitted because it is extremely similar to that of Theorem 6 .

Example 3.6. Suppose both the insurer and the insured use the exponential utility function with index $a$ and $b$, respectively. From Theorem 7 we get the following optimal dividend payments rule:

$$
R^{*}=\left\langle\frac{a}{a+b}(P-X+d)\right\rangle_{U L},
$$

where $d$ is such that $\mathbb{E} \exp \left(a\left(X+R^{*}-M-P\right)\right)=1-a q$. If $L=0$ and if $U=(P-X)_{+}$ then

$$
R^{*}=\min \left\{(P-X)_{+}, \frac{a}{a+b}(P-X+d)_{+}\right\} .
$$

The later result is due to Vandebroek (1988).

\subsection{Optimal limited reinsurance}

Assume that an insurer is exposed to a risk $X$ and has decided to buy a reinsurance policy. By $R$ we denote the part of $X$ covered by reinsurer. The following reinsurance contracts on the basis of global claims are commonly treated in actuarial literature:

stop loss $R=(X-b)_{+}$,

quota share $R=a X$,

combined stop loss and quota share $R=a(X-b)_{+}$,

where $a, b$ are parameters such that $0<a \leq 1$ and $b \geq 0$. We can find several papers on optimality of agreements 1-3 (see e.g. Gerber, 1979, Deprez and Gerber, 1985, Pesonen, 1984, Samson, 1986, Hesselager, 1990, Hesselager, 1993, Bühlmann, 1996, Embrechts et al. 1997, Rolski et al., 1999, Young, 1999, Kaluszka, 2001, 2004, and the references therein). Nevertheless, a lot of these results are only of theoretical value since in practice most insurance is sold with limited liability. Such an insurance contract is the limited stop loss defined by $R=\min \left\{l,(X-b)_{+}\right\}$, where $l$ is a positive real, called the layer (cf. Gerathewohl, 1980, Daykin et al., 1994, Barile and Monti, 1995). It seems that there are only few papers dealing with optimality of limited reinsurance contracts (see Sundt, 1991, Walhin and Paris, 2000, and Mata, 2000). 
We now adjust some well-known optimality results to the case of limited reinsurance. Suppose the cedent wants to minimize the variance of his part of the total claims under a fixed reinsurer's premium based on the mean and the variance of the compensated part of the risk, i.e.

$$
\min \mathbb{D}^{2}(X-R) \text { s.t. } \mathbb{E} R=f(P, \mathbb{D} R), L \leq R \leq U,
$$

where $0 \leq L \leq U \leq X$ are given constraints and $t \rightarrow f(P, t)$ is decreasing and concave function such that $f(P, 0)=P$. Examples include the

- standard deviation principle $P=\mathbb{E} R+\beta \mathbb{D} R$,

- variance principle

- mixed principle

- modified variance principle

- mean value principle

- quadratic utility principle

$$
P=\mathbb{E} R+\beta \mathbb{D}^{2} R,
$$$$
P=\mathbb{E} R+\alpha \mathbb{D} R+\beta \mathbb{D}^{2} R,
$$$$
P=\mathbb{E} R+\beta \mathbb{D}^{2} R / \mathbb{E} R,
$$$$
P=\left[\mathbb{E}\left(R^{2}\right)\right]^{1 / 2}=\left[(\mathbb{E} R)^{2}+\mathbb{D}^{2} R\right]^{1 / 2},
$$$$
P=\mathbb{E} R+c-\left(c^{2}-\mathbb{D}^{2} R\right)^{1 / 2},
$$

where $\alpha, \beta, c>0$ (cf. Gerber, 1979, Goovaerts et al., 1984, and Bühlmann, 1996 among others). Theorem 8 extends Theorem 1.1 of Kaluszka (2001).

\section{Theorem 8.}

Assume $\mathbb{E} X^{2}<\infty$ and assume there exists the rule $R^{*}=\langle a(X-b)\rangle_{U L}$ with reals $a, b$ such that $\mathbb{D} R^{*}>0, a>0$, and

(i) $\left(a(\mathbb{E} X-b)-\mathbb{E} R^{*}\right) f_{2}^{\prime}\left(P, \mathbb{D} R^{*}\right)=(1-a) \mathbb{D} R^{*}$,

(ii) $\mathbb{E} R^{*}=f\left(P, \mathbb{D} R^{*}\right)$,

where $f_{2}^{\prime}(P, t)=\partial f(P, t) / \partial t$. Then $R^{*}$ is a solution of $(3.21)$.

Proof. Put $n=2, R_{1}=R, R_{2}=X-R, L_{1}=L, U_{1}=U, \Pi=-\mathbb{D}^{2} R_{2}, P_{1}=0$, and $H_{1}(R)=f(P, \mathbb{D} R)-\mathbb{E} R$ in Theorem 1 (a) (see Remark 2.2). As a consequence of the inequalities:

$$
\begin{gathered}
f(P, \mathbb{D} \hat{R}) \leq f(P, \mathbb{D} R)+f_{2}^{\prime}(P, \mathbb{D} R)(\mathbb{D} \hat{R}-\mathbb{D} R), \\
\mathbb{D} \hat{R}-\mathbb{D} R \geq \mathbb{E}[(R-\mathbb{E} R)(\hat{R}-R)] / \mathbb{D} R,
\end{gathered}
$$

and the assumption $f_{2}^{\prime}(P, t) \leq 0$, we get

$$
H_{1}(\hat{R}) \leq \begin{cases}H_{1}(R)+\mathbb{E}\left[\left(\frac{R-\mathbb{E} R}{\mathbb{D} R} f_{2}^{\prime}(P, \mathbb{D} R)-1\right)(\hat{R}-R)\right] & \text { if } \mathbb{D} R>0 \\ H_{1}(R)+\mathbb{E}[-(\hat{R}-R)] & \text { if } \mathbb{D} R=0,\end{cases}
$$

Hence $H_{1}$ is a C-functional and

$$
H_{1, R}^{\prime}=\frac{R-\mathbb{E} R}{\mathbb{D} R} f_{2}^{\prime}(P, \mathbb{D} R)-1
$$


provided $\mathbb{D} R>0$ and $H_{1, R}^{\prime}=-1$ otherwise. Moreover, $\Pi$ is a C-functional, $\Pi_{1}^{\prime}=0$, and $\Pi_{2}^{\prime}=-2\left(R_{2}-\mathbb{E} R_{2}\right)=-2(X-R-\mathbb{E}(X-R))$. If $\mathbb{D} R>0$ then the extended Bühlmann-Jewell condition reads

$$
\begin{array}{ll}
2(X-R-\mathbb{E}(X-R))=c\left(1-f_{2}^{\prime}(P, \mathbb{D} R) \frac{R-\mathbb{E} R}{\mathbb{D} R}\right) & \text { if } L<R<U, \\
2(X-R-\mathbb{E}(X-R)) \leq c\left(1-f_{2}^{\prime}(P, \mathbb{D} R) \frac{R-\mathbb{E} R}{\mathbb{D} R}\right) & \text { if } R=L, \\
2(X-R-\mathbb{E}(X-R)) \geq c\left(1-f_{2}^{\prime}(P, \mathbb{D} R) \frac{R-\mathbb{E} R}{\mathbb{D} R}\right) & \text { if } R=U .
\end{array}
$$

Clearly, $R=a(X-b)$ for $L<R<U$ with

$$
a=\left(1-\frac{c}{2} \frac{f_{2}^{\prime}(P, \mathbb{D} R)}{\mathbb{D} R}\right)^{-1}>0, b=\mathbb{E}(X-R)+\frac{c}{2}\left(1+\frac{f_{2}^{\prime}(P, \mathbb{D} R)}{\mathbb{D} R} \mathbb{E} R\right) .
$$

It is easy to check that $(a(\mathbb{E} X-b)-\mathbb{E} R) f_{2}^{\prime}(P, \mathbb{D} R)=(1-a) \mathbb{D} R$. The rule $R^{*}=$ $\langle a(X-b)\rangle_{U L}$ satisfies the extended Bühlmann-Jewell condition, which completes the proof.

We now treat in details the case of the variance principle, namely, we find a solution of the problem

$$
\min \mathbb{D}^{2}(X-R) \text { s.t. } P=\mathbb{E} R+\beta \mathbb{D}^{2} R, L \leq R \leq U,
$$

where $0 \leq L \leq U \leq X$.

\section{Theorem 9.}

Let $\mathbb{P}(a \leq X \leq b)>0$ for every $0<a<b$, let $\mathbb{P}(U<X)>0$, and let $\mathbb{E} L+\beta \mathbb{D}^{2} L<$ $P<\mathbb{E} U+\beta \mathbb{D}^{2} U$. If $0<\beta \leq 1 /(2 \mathbb{E} X)$, then a solution of (3.22) is given by $R_{a b}=$ $\langle a(X-b)\rangle_{U L}$, where $a>0, b \in \mathbb{R}$ solves the following system of equations

$$
a-2 a \beta(\mathbb{E} X-b)+2 \beta \mathbb{E} R_{a b}=1, \quad P=\mathbb{E} R_{a b}+\beta \mathbb{D}^{2} R_{a b}
$$

Proof. The result follows directly from Theorem 8 . It is enough to show that the system (3.23) has a solution. Define $\varphi(a, b)=\mathbb{E} R_{a b}+\beta \mathbb{D}^{2} R_{a b}-P$ for $a>0$, $b \in \mathbb{R}$. Observe that both the function $a \rightarrow \mathbb{E} R_{a b}$ and $a \rightarrow \mathbb{E} R_{a b}^{2}$ are nondecreasing on $(0, \infty)$ for each $b$. Since $\mathbb{E} R_{a b} \leq \mathbb{E} X \leq 1 /(2 \beta)$, the function $a \rightarrow \varphi(a, b)$ is nondecreasing for each $b$. It is clear that $\varphi(0+, b)=\mathbb{E} L+\beta \mathbb{D}^{2} L-P<0$ and

$$
\varphi(\infty, b)= \begin{cases}\mathbb{E} U+\beta \mathbb{D}^{2} U-P>0 \text { for } b \leq 0 \\ \mathbb{E} R_{b}+\beta \mathbb{D}^{2} R_{b}-P \quad \text { for } b>0,\end{cases}
$$

where $R_{b}(x)=L(x)$ for $x<b$ and $R_{b}(x)=U(x)$ for $x \geq b$. Let $c$ denote the smallest solution of the equation $\mathbb{E} R_{b}+\beta \mathbb{D}^{2} R_{b}=P$ in $b>0$. By the assumptions on $X, U$ and $L$, for each $b<c$ the function $a \rightarrow \varphi(a, b)$ is constant on $\left(0, a_{0}\right) \cup$ $\left[a_{1}, \infty\right)$ and is increasing on $\left[a_{0}, a_{1}\right)$ for some $0 \leq a_{0}<a_{1} \leq \infty$ (either the interval 
$\left(0, a_{0}\right)$ or $\left[a_{1}, \infty\right)$ may be empty). Since $\varphi$ is continuous on $(0, \infty) \times \mathbb{R}$, there exists a continuous positive function on $(-\infty, c)$, say $a(b)$, such that $\varphi(a(b), b)=0$ for each $b<c$. Note that $a(-\infty)=0$ because of

$$
\varphi(a,-\infty)=\mathbb{E} U+\beta \mathbb{D} U-P>0
$$

for each $a$. Furthermore, $a(c-)=\infty$. Let $\psi(a, b)=a-2 a \beta(\mathbb{E} X-b)+2 \beta \mathbb{E} R_{a b}-1$. The function $\psi(a(b), b)$ is continuous on $(-\infty, c)$ since both $a(b)$ and $\psi$ are continuous. Observe that

$$
\begin{aligned}
& \lim _{b \rightarrow-\infty} \psi(a(b), b) \leq \lim _{b \rightarrow-\infty}\left(2 \beta \mathbb{E} R_{a b}-1\right) \leq 2 \beta \mathbb{E} U-1<2 \beta \mathbb{E} X-1 \leq 0, \\
& \lim _{b \rightarrow c^{-}} \psi(a(b), b) \geq \lim _{b \rightarrow c^{-}} a(b)(1-2 \beta \mathbb{E} X+2 \beta b)-1=\infty,
\end{aligned}
$$

because $a(-\infty)=0, a(c-)=\infty, 1-2 \beta \mathbb{E} X \geq 0$, and $c>0$. Therefore, there exist $a>0$ and $b \in \mathbb{R}$ such that $a=a(b), \varphi(a, b)=0$, and $\psi(a, b)=0$, which proves the desired result.

We now propose another solution of the problem of incorporating the variance of reinsurer's cover into a reinsurance contract. Suppose the reinsurer uses the expected value principle. Both sides of the contract accept a rule being a solution of the problem

$$
\min \mathbb{D}^{2}(X-R)+k \mathbb{D}^{2} R \text { s.t. } P=(1+\beta) \mathbb{E} R, L \leq R \leq U,
$$

where $0 \leq L \leq U \leq X$ and $k>0$ is a parameter which has been chosen by one of the methods described in Section 1.

\section{Theorem 10.}

Suppose $(1+\beta) \mathbb{E} L<P<(1+\beta) \mathbb{E} U$. Then a solution of (3.24) is the limited combination of a stop loss and quota share given by $R^{*}=\left\langle\frac{1}{k+1}(X-b)\right\rangle_{U L}$, where $b$ is the solution of $(1+\beta) \mathbb{E} R^{*}=P$.

Proof. Directly from Theorem 1 (a) and Remark 2.2 with $n=2, R_{1}=R, R_{2}=$ $X-R, \Pi=-k \mathbb{D}^{2} R_{1}-\mathbb{D}^{2} R_{2}, H_{1}\left(R_{1}\right)=(1+\beta) \mathbb{E} R_{1}$, and $P_{1}=P$.

\section{ACKNOWLEDGMENTS}

The author would like to thank the referees for their valuable comments which led to improvements in the paper.

\section{REFERENCES}

AAsE, K.K. (1993) Equilibrium in a reinsurance syndicate; existence, uniqueness and characterization. ASTIN Bulletin 23, 185-211.

Ammeter, H., Depoid, P. and De FinetTi, B. (1959) L'étude matématique des assurances non viagères dans l'europe continentale occidentale. ASTIN Bulletin 1, 46-70. 
Arrow, K.J. (1963) Uncertainty and the welfare economics of medical care. The American Economic Review 53, 941-73.

Barile, A. and Monti, R.G. (1995) A practical guide to finite risk insurance and reinsurance. John Wiley. New York.

Borch, K. (1962) Equilibrium in a reinsurance market. Econometrica 30, 424-444.

Borch, K. (1974) The Mathematical Theory of Insurance. Lexington Books, Lexington.

BorCH, K. (1990) Economics of Insurance. Edited by K.K. Aase and A. Sandmo, North-Holland. Amsterdam.

BÜHLmanN, H. (1984) The general economic premium principle. ASTIN Bulletin 14, 13-21.

Bühlmann, H. (1996) Mathematical models in risk theory. 2nd edition, Springer-Verlag, Berlin.

BühlmanN, H. and Jewell, W.S. (1979) Optimal risk exchanges. ASTIN Bulletin 10, 243-262.

Daykin, C.D., Pentikäinen, T. and Pesonen, M. (1994) Practical Risk Theory for Actuaries. Chapman \& Hall, London.

De FinetTi, B. (1940) Il problema dei pieni. Giorn. Ist. Ital. Attuari 11, 1-88.

DePreZ, O. and Gerber, H.U. (1985) On convex principles of premium calculation. Insurance: Mathematics \& Economics 4, 179-189.

Embrechts, P., KlüPpelberg, C. and Mikosch, T. (1997) Modelling Extremal Events. SpringerVerlag, Berlin.

Gerathewohl, K. (1980) Reinsurance Principles and Practice. Verlag Versicherungswirtschaft, Karlsruhe.

GERBER, H.U. and Jones, D.A. (1974) Dividend formulas in group insurance. Transactions of the Society of Actuaries 26, 77-86.

Gerber, H.U. (1978) Pareto-optimal risk exchanges and related decision problems. ASTIN Bulletin 9, 25-33.

Gerber, H.U. (1979) An Introduction to Mathematical Risk Theory. S.S. Huebner Foundation for Insurance Education, Wharton School, University of Pennsylvania, Philadelphia.

Gerber, H.U. and Pafumi, G. (1999) Utility functions: from risk theory to finance. North American Actuarial Journal 2, 74-100.

Gollier, Ch. (2001) The Economics of Risk and Time, The MIT Press, Cambridge.

Goovaerts, M.J., De Vylder, F. and Haezendonck, J. (1984) Insurance Premiums: Theory and Applications. North-Holland, Amsterdam.

Hesselager, O. (1990) Some results on optimal reinsurance in terms of the adjustment coefficient. Scandinavian Actuarial Journal, 80-95.

Hesselager, O. (1993) Extensions of Ohlin's lemma with applications to optimal reinsurance structures. Insurance: Mathematics \& Economics 13, 83-97.

KALUSZKA, M. (2001) Optimal reinsurance under mean-variance premium principles. Insurance: Mathematics \& Economics 28, 61-67.

KALUSZKA, M. (2004) Mean-variance optimal reinsurance arrangements. Scandinavian Actuarial Journal (to appear)

LemaiRe, J. (1991) Cooperative game theory and its insurance applications. ASTIN Bulletin 21, $17-40$

Lemaire, J. and Quairiere, J.P. (1986) Chains of reinsurance revisited. ASTIN Bulletin 16, 7788

LiENHARD, M. (1986) Calculation of price equilibria for utility functions of the Hara class. ASTIN Bulletin 16, 91-98.

MatA, A.J. (2000) Pricing excess of loss reinsurance with reinstatements. ASTIN Bulletin 30, 349-368.

Panjer, H. (editor), Boyle, P., Cox, S., Dufresne, D., Gerber, H., Mueller, H., Pedersen, H., Pliska, S., Sherris, M., Shiu, E. and TAn, K. (1998) Financial Economics With Applications to Investments, Insurance and Pensions. Schaumburg, III., The Actuarial Foundation.

Pesonen, M.I. (1984) Optimal reinsurances. Scandinavian Actuarial Journal, 65-90.

Rockafellar, R.T. (1970) Convex Analysis. Princeton University Press, Princeton. New Jersey.

Rolski, T., Schmidli, H., Schmidt, V. and Teugels, J. (1999) Stochastic Processes for Insurance and Finance. J. Wiley \& Sons, Chichester.

SAMSON, D. (1986) Expected utility strategic decision models for general insurers. ASTIN Bulletin 16, 45-58. 
Sundt, B. (1991) On excess of loss reinsurance with reinstatement. Bulletin of the Swiss Actuaries, 51-66.

VAndebroek, M. (1988) Pareto-optimal profit-sharing. ASTIN Bulletin 18, 47-56.

WALHIN, J.F. and PARIS, J. (2000) The effect of excess-of-loss reinsurance with reinstatements on the cedent's portfolio. Blätter der Deutsche Gesellschaft für Versicherungsmathematik 24, 615627.

WYLER E. (1990) Pareto optimal risk exchanges and a system of differential equations - a duality theorem. ASTIN Bulletin 20, 23-31.

Young, V.R. (1999) Optimal insurance under Wang's premium principle. Insurance: Mathematics \& Economics 25, 109-122.

MareK KALUSZKA

Institute of Mathematics

Technical University of Lodz

Ul. Zwirki 36

90-924 Lodz

Poland

E-mail:kaluszka@p.lodz.pl 